\title{
Morfoanatomia e biometria seminal de cinco espécies de Cattleya Lindl. (Orchidaceae)
}

\author{
Crislâine de Fátima Eggert \& Cristiano Pedroso-de-Moraes
}

Fundação Hermínio Ometto, Uniararas, Jardim Universitário, Av. Dr. Maximiliano Baruto, 500, CEP 13607-339, Araras, São Paulo, Brasil cpmoraes@gmail.com, cris_eggert@yahoo.com.br

Recebido em 18.X.2013

Aceito em 8.XII.2017

DOI 10.21826/2446-8231201772314

RESUMO - Nas espécies Cattleya bicolor Lindl., C. loddigesii Lindl., C. lundii (Rchb. f. \&Warm.) Van den Berg, C. purpurata Lindl.e C. tigrina A. Rich, existem dificuldades de delimitação morfológica e incertezas quanto a sua taxonomia. Este estudo teve por objetivo caracterizar morfobiometricamente sementes das cinco espécies no intuito de apontar características úteis à taxonomia do gênero. Sementes maduras oriundas de autopolinizações manuais foram coletadas de cápsulas em início de deiscência e submetidas à desidratação em série etílica, coloração com a mistura de safranina e azul de astra modificada e analisadas em microscopia óptica. Os resultados demonstraram que $C$. lundii difere das demais espécies por apresentar coloração seminal amarelo-ouro. A análise apontou semelhanças morfológicas entre $C$. loddigesii e $C$. lundii e entre $C$. bicolor e $C$. tigrina. Os caracteres morfológicos analisados são muito semelhantes entre as espécies.

Palavras-chave: Laeliinae, orquídea, semente

\begin{abstract}
Seed morphoanatomy and biometrics of five species of Cattleya Lindl. (Orchidaceae). In the species Cattleya bicolor Lindl., C. loddigesii Lindl., C. lundii (Rchb. f. \&Warm.) Van den Berg, C. purpurata Lindl. and C. tigrina A. Rich, there are difficulties in morphological delimitation and uncertainties about their taxonomy. This study aimed to characterize morphobiometrically seeds of five species in order to highlight useful characteristics for the taxonomy of the genus. Mature seeds derived from artificial self-pollination were collected from capsules in early dehiscence and subjected to dehydration in ethanol series, stained with a mixture of astra blue and safranin and analyzed by light microscopy. At the end of the tests, we verified that $C$. lundii can be distinguished from other species by yellow gold seeds. The analysis also showed morphological similarities between $C$. loddigesii Lindl. and C. lundii, and between C. bicolor Lindl. and C. tigrina. The morphological characters analyzed are very similar among species.
\end{abstract}

Key-words: Laeliinae, orchid, seed

\section{INTRODUÇÃO}

Características seminais em Orchidaceae são amplamente utilizadas para a elucidação de problemas taxonômicos (Clifford \& Smith 1969, Barthlott \& Ziegler 1981). Várias classificações baseadas em características micromorfológicas foram desenvolvidas para orquídeas (Dressler 1981), pois caracteres seminais servem como critérios na elucidação de questões de ordem taxonômica de grupos genéricos e subgenéricos (Barthlott \& Ziegler 1981, Mathews \& Levins 1986, Ness 1989, Vij et al. 1992, Larry 1995, Augustine et al.2001). As características morfológicas das sementes não somente apresentam relevância taxonômica, mas também podem ser utilizadas para deduzir relações filogenéticas (Barthlott \& Ziegler 1981). Assim, as características biomorfométricas das sementes foram usadas na busca do entendimento filogenético, principalmente, entre representantes das várias subtribos de Orchidaceae (Rani et al. 1993, Augustine et al. 2001).

As sementes de orquídeas variam em tamanho, morfologia, estrutura e cor. O tamanho das sementes pode variar de 150 a $6.000 \mu \mathrm{m}$ e, na maioria dos taxa, o intervalo biométrico encontrado é de 300-800 $\mu \mathrm{m}$. Tais sementes variam amplamente em forma, podendo ser elipsoidais a fusiformes, com frequência clavadas a filiformes e, às vezes, proeminentemente aladas (Molvray \& Kores 1995). Em alguns casos, as sementes podem apresentar-se cobertas por firme tegumento, entretanto, na maioria das sementes da família, esse é extremamente delgado e envolve livremente o embrião, devido à ausência de endosperma. $\mathrm{O}$ número de células que forma a testa varia muito entre as diferentes espécies, podendo chegar até 20 (Molvray \& Kores 1995). As dimensões celulares encontradas podem ser uniformes ou, em algumas, sensivelmente maiores ou menores em áreas como a região mediana ou extremidade calazal (Augustine et al. 2001). As paredes celulares periclinais externas da testa podem ser lisas ou reticuladas; se a reticulação está presente, os padrões podem ser também diversos (Molvray \& Kores 1995).

As sementes de Orchidaceae geralmente não apresentam endosperma, e esse, quando presente, se mostra como uma camada adjacente de carboidratos, transparente e 
indiferenciada ao redor dos embriões (Arditti et al. 1980). Testa e embrião de diferentes taxa de Orchidaceae podem variar com relação à coloração e relações volumétricas (Arditti et al. 1980, Augustine et al. 2001). Geralmente, as sementes de orquídeas são divididas em 20 diferentes tipos, sendo tal tipificação baseada na forma seminal, extensão relativa de células da testa, altura e ornamentação da testa, incluindo a presença da abertura micropilar (Barthlott \& Ziegler 1981, Vij et al. 1992).

São descritas para o Brasil de 64 a 77 espécies de Cattleya Lindl. (Cogniaux 1898, Pabst \& Dungs 1975, Van den Berg 2008). O gênero, na atualidade, apresentase monofilético (Buzatto et al. 2010) com suporte de estudos filogenéticos baseados em dados moleculares, encontrando-se inserido na subfamília Epidendroideae, tribo Epidendreae, subtribo Laeliinae (Van den Berg, 2008, Van den Berg et al. 2009). Em sua delimitação atual, o gênero circunscreve espécies anteriormente inclusas em Eunannos P. Campos Porto \& A.C. Brade, Laelia Lindl. e Sophronitis Lindl., além de espécies segregadas, tais como as pertencentes aos gêneros Hadrolaelia (Schltr.) Chiron \& V. P. Castro, Dungsia Chiron \& V. P. Castro, Hoffmannseggella H. G. Jones, Chironiella Braem, Microlaelia Chiron \& V. P. Castro e Schluckebieria Braem (Van den Berg 2008, Van den Berg et al. 2009, Buzatto et al. 2010). A separação artificial do gênero Cattleya, baseada no número de polínias (oito em Laelia e quatro em Cattleya) (Van den Berg \& Chase 2004), principalmente com relação às espécies brasileiras inseridas em Laelia Lindl. sugeria que as Laelia Lindl. da seção Cattleyodes apresentavam-se mais aparentadas a Cattleya e, portanto, deveriam estar inseridas neste gênero (Dressler 1981, 1993).

Com relação à semente, apenas alguns taxa de orquídeas foram investigados até o presente momento (Vij et al. 1992, Rani et al. 1993, Augustine et al. 2001, Swamy et al. 2004). Portanto, há escassos registros sobre a morfologia de sementes de representantes brasileiros da família, o que demonstra que trabalhos morfoanatômicos e biométricos se tornam extremamente relevantes, devido à necessidade da elucidação de problemas e dúvidas de ordem sistemática nas Orchidaceae brasileiras.

Dessa forma, este estudo teve por objetivo caracterizar morfologicamente e biometricamente, sob microscopia de luz, as sementes de cinco espécies de Cattleya e apontar caracteres passíveis de serem utilizados na taxonomia do gênero.

\section{MATERIAL E MÉTODOS}

\section{Obtenção das sementes}

A escolha das espécies ocorreu devido à necessidade de se obter caracteres seminais passíveis de serem utilizados na identificação de plantas tais como C. bicolor e C. tigrina, as quais já chegaram a ser descritas como uma única espécie (Moraes 2014); à dificuldade da realização de rápida diferenciação entre $C$. loddigesii e $C$. harrisoniana (Pedroso-de-Moraes 2000); e por C. purpurata e $C$. lundii terem pertencido anteriormente ao gênero Laelia, posteriormente aos gêneros Hadrolaelia e Microlaelia, respectivamente e, atualmente encontrarem-se inseridas em Cattleya (Buzatto et al. 2010), existindo ainda incertezas sobre sua inclusão nesse gênero.

Sementes maduras das cinco espécies estudadas, oriundas de autopolinizações manuais, forma mais usual de obtenção de sementes em experimentos controlados (Pansarin, 2003), foram coletadas de cápsulas em início de deiscência nos anos de 2010-2012 no Orquidário do Centro Universitário Hermínio Ometto, Uniararas, Araras, São Paulo, Brasil.

As sementes coletadas de quatro diferentes indivíduos por espécie foram homogeneizadas para o início dos trabalhos quali-quantitativos e biométricos.

Primeiramente, foi realizada a contagem, separação e pesagem de mil sementes por espécie em balança analítica e a determinação da coloração apresentada pelas sementes e embriões, onde parte das amostras foram depositadas em vidros de relógio e analisadas sob aumento de 40x em estereomicroscópio OpTech, MD30. A determinação da coloração dos embriões foi propiciada pela transparência da testa (Swamy et al. 2004).

\section{Análises anatômicas e biométricas das sementes}

Para as análises anatômicas e biométricas, as sementes foram fixadas em solução de FAA (Johansen 1940) e preservados em álcool 70\%. Essas foram desidratadas em série etílica e, posteriormente, coradas com mistura de safranina e azul de astra (Bukatsh 1972, modificado para $0,5 \%$ ) e montadas em bálsamo do Canadá. Para a identificação de compostos químicos, utilizou-se a solução de lugol para amido (Johansen 1940), de floroglucina clorídrica para lignina (Jensen 1962), e de sudan III para lipídios (Johansen 1940).

Para cada espécie analisada, foram preparadas quatro lâminas, contendo 100 sementes cada, totalizando 400 análises biométricas por espécie. A largura e o comprimento das sementes foram avaliados. Foi auferida a proporção comprimento/largura, porcentagem de sementes com embriões e as médias do comprimento. Também foi analisada a largura celular média, o número médio de células da testa e a porcentagem de espaço aerífero evidenciado em 400 sementes de cada espécie (Swamy et al. 2004). Foram também descritos os padrões de ornamentação do tegumento, isto é, presença ou ausência de reticulação e presença de abertura na extremidade micropilar. $\mathrm{O}$ volume das sementes foi calculado usando a fórmula $2\left[(\mathrm{~L} / 2)^{2}(1 / 2 \mathrm{C})\right.$ (1.047)], onde $\mathrm{L}=$ largura da semente, $\mathrm{C}=$ comprimento da semente, $1.047=\pi /{ }_{3}$ (Arditti et al., 1980).

\section{Análises anatômicas e biométricas dos embriões}

Para os embriões, foram realizadas medições concernentes ao comprimento e largura. Os embriões das orquídeas são elípticos, portanto seu volume foi calculado usando a fórmula $4 / 3 \pi \mathrm{ab}^{2}$, onde $\mathrm{a}=1 / 2 \mathrm{X}$ comprimento e $\mathrm{b}=1 / 2 \mathrm{X}$ largura do embrião (Swamy et al. 2004). Após esse cálculo, pode-se auferir a proporção volume seminal/ 
volume embrionário (Swamy et al. 2004). Os resultados foram registrados pela utilização de fotomicroscópio Leica DM 2000 e uso do software IM 50.

Todas as análises biométricas foram realizadas com o auxílio do programa AnatiQuanti 2.0. Os dados obtidos foram submetidos à análise de variância (ANOVA) e comparação entre as médias pelo teste de Tukey a $1 \%$ de probabilidade (Sokal \& Rohlf 1981, Santana \& Ranal 2004), pela utilização do aplicativo estatístico BioEstat 5.3.

\section{RESULTADOS}

\section{Análises anatômicas e biométricas das sementes}

As análises qualitativas realizadas revelaram que as mil sementes de cada uma das espécies analisadas apresentam coloração amarelo-claro, exceto Cattleya lundii, com coloração amarelo-ouro. Na análise morfológica seminal, pode-se constatar formato filiforme para $C$. loddigesii (Fig. 1A), elíptico-afilada para C. lundii (Fig. 1B) e fusiforme para C. bicolor, C. purpurata e C. tigrina (Figs. 1C-E, G). Todas as espécies apresentaram reticulações nas paredes celulares periclinais externas, que compõem a epiderme uniestratificada da testa, sendo que, em C. bicolor, $C$. loddigesii e $C$. tigrina, pode-se visualizar facilmente a abertura micropilar (Fig. 1D). Em todas as espécies, a extremidade calazal apresenta-se afunilada (Fig. 1).

Com relação às análises quantitativas, para a variável peso de 1.000 sementes, C. tigrina, C. lundii e C. loddigesii apresentaram-se como as mais pesadas, enquanto $C$. bicolor assumiu valor mediano para a variável e C. purpurata caracterizou-se como a mais leve (Tab. 1). Para a porcentagem de sementes com embriões, os resultados obtidos demonstraram que $C$. lundii e $C$. loddigesii possuem maior porcentagem de sementes com embriões. Entretanto, em C. purpurata, os baixos percentuais obtidos relacionamse ao fato de que a maioria de suas sementes apresentaram embriões abortados (Tab. 1, Fig. 1E).

As espécies C. loddigesii, C. lundii e C. purpurata apresentaram a maior relação comprimento/largura em detrimento de $C$. bicolor, que apresentou o menor valor médio para a variável (Tab. 1). Os maiores volumes médios encontrados nas sementes ocorreram nas espécies $C$. bicolor, $C$. lundii e C. tigrina, enquanto $C$. purpurata apresentou o menor volume médio (Tab. 1).

Com relação ao comprimento celular da testa, $C$. lundii apresentou o maior valor médio, enquanto C. purpurata, o menor (Tab. 1). A maior média da largura celular da testa foi obtida para as espécies C. bicolor, C. purpurata e C. tigrina, as quais apresentaram o mesmo valor numérico médio após a realização da ANOVA, sendo que C. loddigesii obteve o menor valor médio (Tab. 1). Para o número de células da testa, $C$. loddigesii e $C$. lundii apresentaram o maior valor médio, enquanto $C$. tigrina apresentou o menor (Tab. 1). A análise da porcentagem do espaço aerífero das sementes revelou maiores valores médios para C. bicolor, C. lundii, C. tigrina em detrimento de C. loddigesii e $C$. purpurata (Tab. 1).

\section{Análises anatômicas e biométricas dos embriões}

A observação microscópica dos embriões evidenciou uniformidade cromática, sendo observada coloração amarelo-claro em todas as espécies analisadas. De modo geral, todos os embriões caracterizaram-se por serem formados por massas celulares indiferenciadas. C. bicolor, C. purpurata e C. tigrina (Fig. 1G, Tab. 2) apresentaram morfologia embrionária esférica, enquanto $C$. loddigesii e C. lundii demonstraram formatos elipsoides (Fig. 1H, Tab. 2).

As análises biométricas demonstraram que, para a variável comprimento embrionário, o maior valor médio obtido foi para $C$. lundii, enquanto o menor foi encontrado para C. purpurata (Tab. 2). Com relação à largura dos embriões, pode-se observar uniformidade numérica em C. bicolor, C. loddigesii, C. lundii e C. tigrina, enquanto C. purpurata assumiu os menores valores médios para a variável (Tab. 2). A espécie C. purpurata apresentou o maior valor médio para a variável relação comprimento/largura, enquanto $C$. bicolor e C. tigrina obtiveram os menores valores. A variável volume embrionário não apresentou resultados estatísticos capazes de diferenciar as espécies analisadas. Entretanto, para a variável relação volume da semente/embrião, pode-se observar que $C$. bicolor e $C$. tigrina assumiram os maiores valores médios, enquanto C. loddigesii e C. lundii, obtiveram os menores (Tab. 2).

\section{DISCUSSÃO}

\section{Análises anatômicas e biométricas das sementes}

As cores das sementes em Orchidaceae variam de branco, como encontrado em Eria dalzelli (Dalz.) Lindl. (Swamy et al. 2004) a preto, como em Vanilla planifolia Jacks ex. Andr. (Correa 1984, Pedroso-deMoraes \& Fontana 2005, Pedroso-de-Moraes et al. 2012). Entretanto, colorações amarelas, com variações cromáticas de intensidade, são as mais comumente relatadas na literatura para Bletiinae, Chysinae, Coeliinae, Laeliinae, Pleurothallidinae e Ponerinae, pertencentes à Epidendreae (Pridgeon et al. 2006).

Coloração amarelo-clara, como a encontrada em Cattleya bicolor, C. loddigesii, C. purpurata e C. tigrina também foi reportada para Coelogyne breviscapa Lindl. (Swamy et al. 2004). A coloração amarelo-ouro, descrita neste trabalho para Cattleya lundii, também foi evidenciada em Bulbophyllum mysorense (Rolfe) J.J. Smith (Swamy et al. 2004).

Em relação à testa, todas as Cattleya analisadas apresentaram um padrão morfoanatômico similar ao encontrado para as espécies do gênero e para a maioria das Orchidaceae (Barthlott \& Ziegler 1981, Jeeja \& Aansari 1994), ou seja, células da testa delgadas.

As sementes de orquídeas podem ser divididas em 21 tipos morfológicos básicos, sendo tal classificação resultante de uma combinação de caracteres concernentes à forma, número de células, comprimento e ornamentação das células da testa e a presença de orifícios (Dressler, 1993). As sementes 

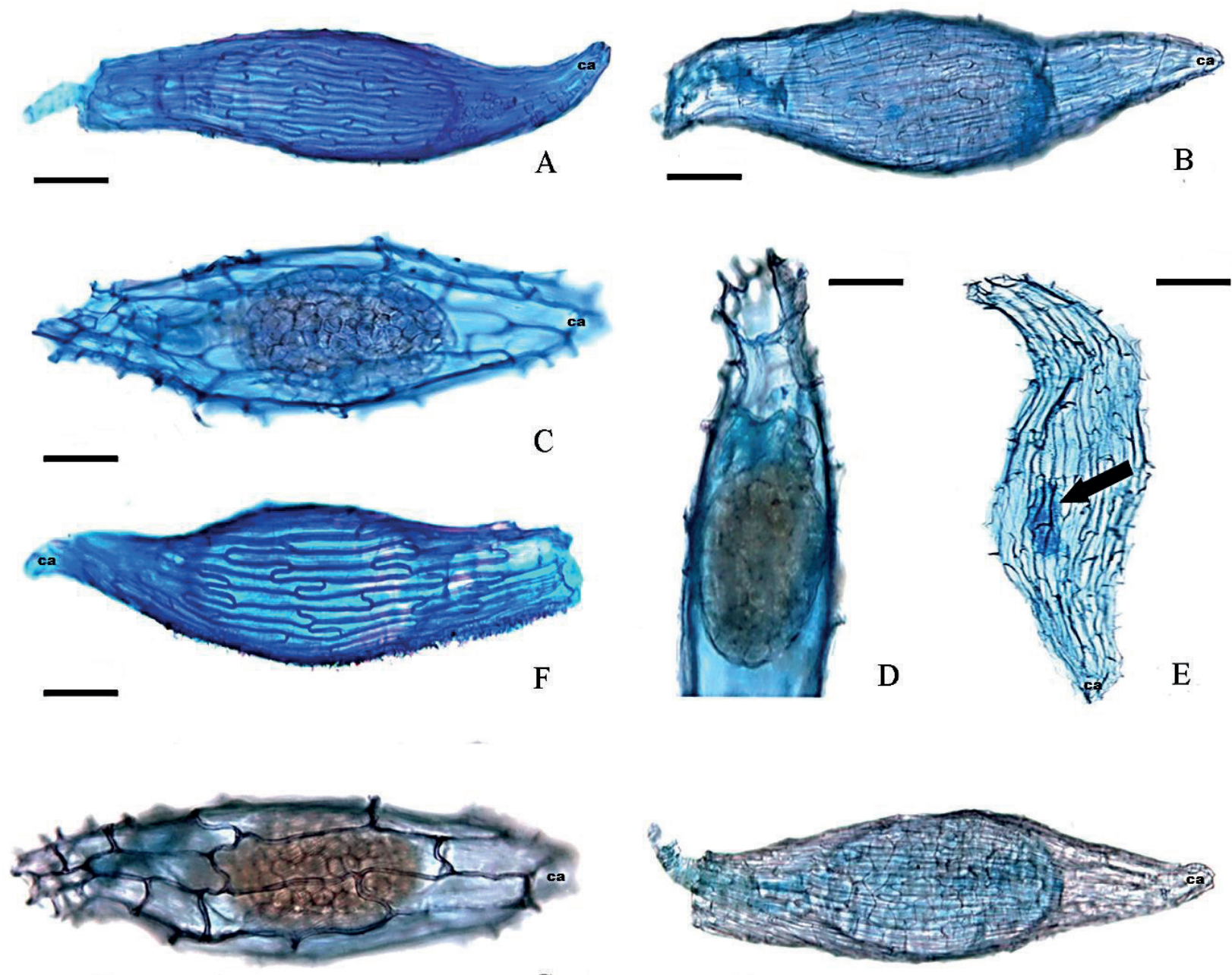

G

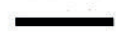

$\mathrm{H}$
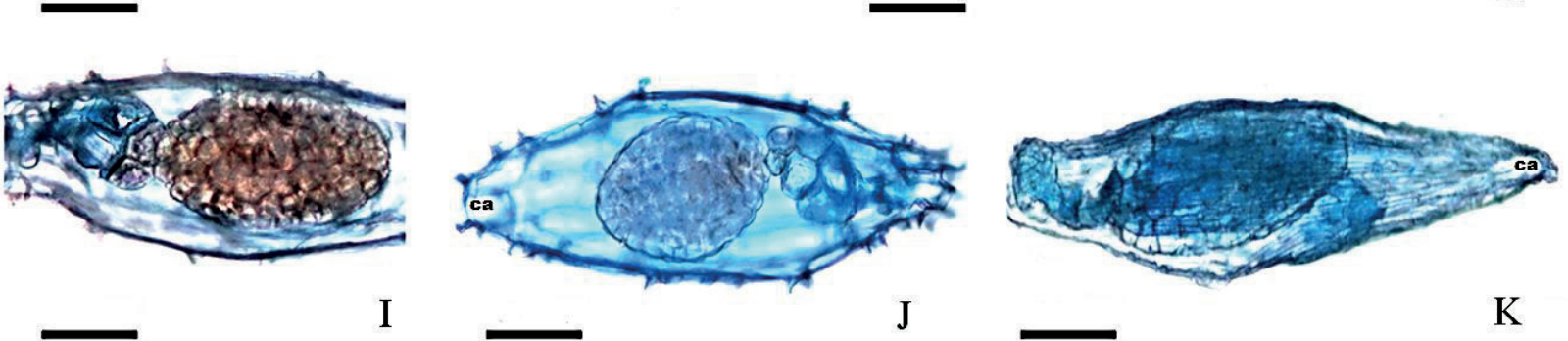

Figs. 1 A-K. A. Semente filiforme de Cattleya loddigesii; B. Semente elíptico-afilada de Cattleya lundii; C. Semente fusiforme de Cattleya bicolor; D. Abertura micropilar da semente de C. bicolor; E. Embrião abortado (seta) contido na semente de Cattleya purpurata; F. Semente evidenciando células superficiais da testa de C.loddigesii; G. Semente evidenciando células da testa e embrião de Cattleya tigrina; H. Semente com embrião elipsóide de C. lundii; I-K. Embriões com suspensores de C. tigrina, C. bicolor e C. lundii (ca = calaza). Barras $=50 \mu \mathrm{m}$.

de Cattleya analisadas neste estudo são classificadas como do tipo Cymbidium, devido a tais sementes serem providas de espaços aeríferos internos, embriões amarelos visíveis através da testa e células tegumentares apresentando-se poligonais e de paredes celulares periclinais externas reticuladas (Barthlott \& Ziegler 1981), corroborando dados moleculares que inserem Laellinae Benth. em Epidendreae Kunth, Epidendroideae Lindl. (Van den Berg \& Chase 2000, Van den Berg et al. 2000, Van den Berg \& Chase 2004, Pridgeon et al. 2006, Van den Berg 2008, Van den Berg et al. 2009). Além disso, duas das espécies analisadas,
C. lundii e C. purpurata, que anteriormente estavam circunscritas em Laelia Lindl., neste trabalho apresentam caracteres morfológicos semelhantes aos das demais espécies comparadas, classicamente pertencentes ao gênero Cattleya Lindl. corroborando resultados morfológicos e moleculares relacionados à filogenia (Dressler 1981, 1993, Van den Berg 2008, Van den Berg et al. 2009).

Quanto ao formato propriamente dito das sementes, morfologias fusiformes, como as encontradas para Cattleya. bicolor, C. purpurata e C. tigrina, e elipsóides são as mais comumente descritas em Orchidaceae (Chase \& 


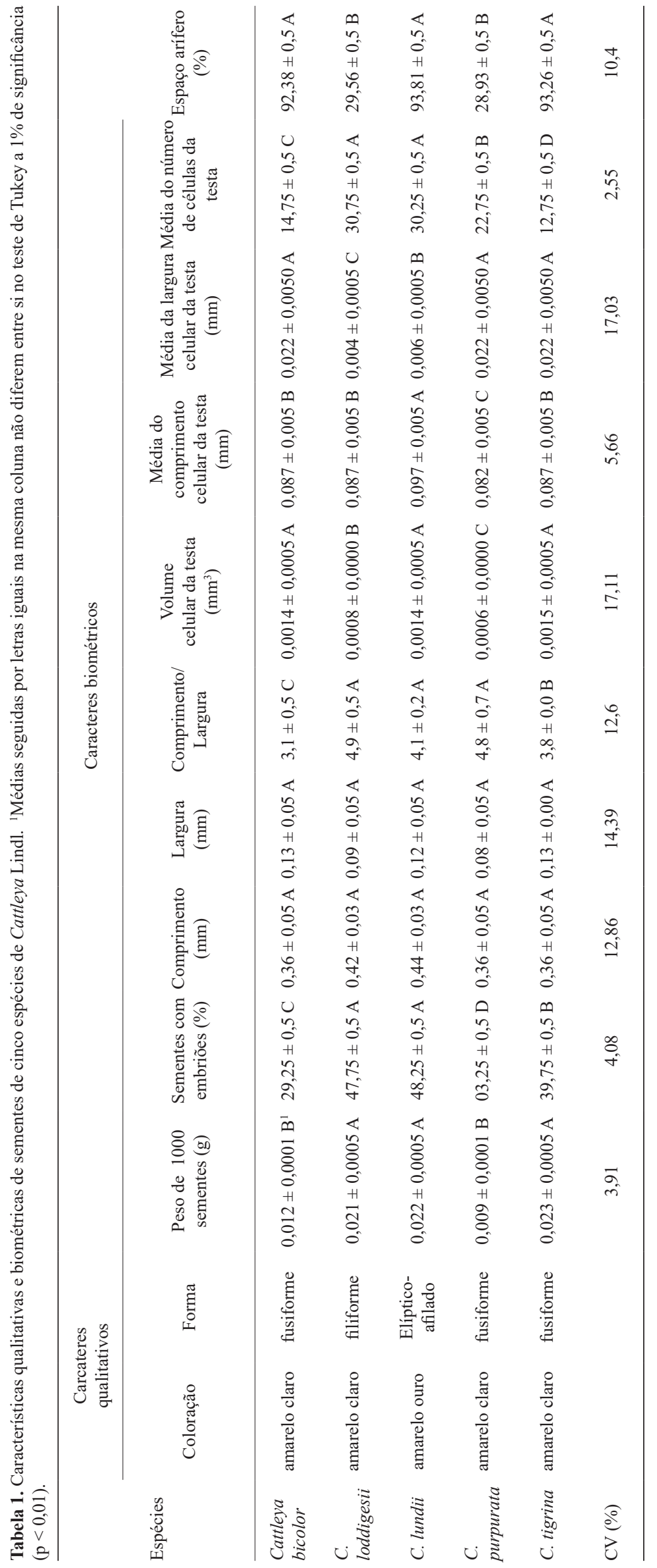


Tabela 2. Características qualitativas e biométricas de embriões de cinco espécies de Cattleya Lindl. ${ }^{1}$ Médias seguidas por letras iguais na mesma coluna não diferem entre si no teste de Tukey a $1 \%$ de significância $(\mathrm{p}<0,01)$

\begin{tabular}{|c|c|c|c|c|c|c|c|}
\hline \multirow[b]{2}{*}{ Espécies } & \multicolumn{7}{|c|}{ Carcateres qualitativos } \\
\hline & Coloração & Formato & $\begin{array}{l}\text { Comprimento } \\
(\mathrm{mm})\end{array}$ & $\begin{array}{l}\text { Largura } \\
(\mathrm{mm})\end{array}$ & $\begin{array}{l}\text { Comprimento/ } \\
\text { Largura }\end{array}$ & $\begin{array}{l}\text { Volume } \\
\left(\mathrm{mm}^{3}\right)\end{array}$ & $\begin{array}{c}\text { Proporção vo- } \\
\text { lume seminal/ } \\
\text { embrionário }\end{array}$ \\
\hline $\begin{array}{l}\text { Cattleya } \\
\text { bicolor }\end{array}$ & amarelo-claro & esférico & $0,12 \pm 0,05 \mathrm{~B}^{1}$ & $0,08 \pm 0,02 \mathrm{~A}$ & $1,4 \pm 0,4 \mathrm{C}$ & $\begin{array}{l}0,0005 \pm \\
0,0005 \mathrm{~A}\end{array}$ & $3,33 \pm 0,5 \mathrm{~A}$ \\
\hline C. loddigesii & amarelo-claro & elipsóide & $0,18 \pm 0,02 \mathrm{~B}$ & $0,08 \pm 0,02 \mathrm{~A}$ & $2,5 \pm 0,5 \mathrm{~B}$ & $\begin{array}{l}0,0006 \pm \\
0,0005 \mathrm{~A}\end{array}$ & $1,51 \pm 0,5 \mathrm{~B}$ \\
\hline C. lundii & amarelo-claro & elipsóide & $0,22 \pm 0,01 \mathrm{~A}$ & $0,08 \pm 0,01 \mathrm{~A}$ & $2,3 \pm 0,3 \mathrm{~B}$ & $\begin{array}{l}0,0011 \pm \\
0,0005 \mathrm{~A}\end{array}$ & $1,42 \pm 0,5 \mathrm{C}$ \\
\hline C. purpurata & amarelo-claro & esférico & $0,08 \pm 0,01 \mathrm{D}$ & $0,03 \pm 0,02 \mathrm{~B}$ & $3,6 \pm 0,5 \mathrm{~A}$ & $\begin{array}{l}0,0008 \pm \\
0,0005 \mathrm{~A}\end{array}$ & $2,56 \pm 0,5 \mathrm{~B}$ \\
\hline C. tigrina & amarelo-claro & esférico & $0,13 \pm 0,02 \mathrm{C}$ & $0,09 \pm 0,02 \mathrm{~A}$ & $1,4 \pm 0,5 \mathrm{C}$ & $\begin{array}{l}0,0006 \pm \\
0,0005 \mathrm{~A}\end{array}$ & $2,52 \pm 0,5 \mathrm{~A}$ \\
\hline CV $(\%)$ & & & 5,64 & 1,14 & 14,78 & 2,11 & 5,77 \\
\hline
\end{tabular}

Pippen 1990, Augustine et al. 2001, Swamy et al. 2004) em detrimento das filiformes e elíptico-afiladas (Barthlott \& Ziegler 1981), observadas para C. loddigesii e C. lundii, respectivamente. Na literatura, sementes fusiformes foram reportadas para as espécies Cymbidium aloifolium (L.) Sw (Vij et al. 1992) e B. mysorense (Rolfe) J. J. (Swamy et al. 2004) e filiformes para Coelogyne elata Lindl e Cymbidium lancifolium Hook (Vij et al. 1992), todas pertencentes à subfamília Epidendroideae, bem como o gênero Cattleya.

Pesagens são amplamente utilizadas para detecção de vigor seminal, pois quanto mais elevado o peso, maior é a germinabilidade em lotes de sementes (Carneiro 1985, Frazão et al. 1985, Brasil 2009). Contudo, devido às dimensões diminutas de sementes de orquídeas, essa prática não é comumente utilizada, não sendo encontradas na literatura referências passíveis de serem utilizadas de forma comparada. Porém, é válido ressaltar que, em trabalhos relacionados à germinação in vitro, as sementes obtidas por autopolinização das espécies $C$. loddigesii e C. tigrina, mesmo em diferentes meios de cultivo, apresentaram alta germinabilidade, sendo apenas seu posterior desenvolvimento afetado pela composição dos meios (Pedroso-de-Moraes et al. 2009 a, b). Além disso, os maiores valores médios obtidos para o peso de 1.000 sementes relacionam-se diretamente à variável porcentagem de sementes com embriões, nas quais as espécies supracitadas, conjuntamente com $C$. lundii, apresentaram os maiores valores, em virtude do acréscimo da massa embrionária. Tal hipótese é corroborada pelo fato de que $C$. purpurata, que apresentou várias sementes com embriões abortados ou pouco vigorosos, obteve os menores valores médios para as duas variáveis analisadas. Ainda, com relação à baixa porcentagem de sementes sem embriões ou com embriões abortados, estudos relacionados à autopolinização e à polinização cruzada em 15 espécies de Cattleya Lindl. verificaram que, de modo geral, a frequência de sementes com embrião não é alta, mesmo em flores nas quais houve a ocorrência de polinização cruzada (Stort \&
Martins 1980). Tais constatações são, ainda, embasadas por resultados encontrados para outras Laeliinae, como $C$. eldorado Linden., na qual a autopolinização manual gerou baixa taxa de sementes viáveis, na ordem de 28,8\% (Stort et al. 2011); em Epidendrum paniculatum Ruiz \& Pavón, a taxa de frutificação após a autopolinização manual em estufa foi de $1,28 \%$ e em campo foi nula, enquanto que a polinização cruzada, nas mesmas condições, alcançou os percentuais de 95,6\% e 100\%, respectivamente (Pansarin 2003).

Entre as orquídeas, a maioria das espécies é autocompatível; no entanto, a polinização cruzada parece ser a forma mais comum de se obter a fecundação (Pijl \& Dodson 1966, Dressler 1981). A prevenção da autopolinização é favorecida pela presença de mecanismos florais (Pijl \& Dodson 1966, Dressler 1981, Catling \& Catling 1991) e, em grupos mais derivados, por sistemas de auto-incompatibilidade genética como relatado, por exemplo, para Oncidiinae (Pridgeon et al. 2009). Ainda, baixa disponibilidade de nutrientes (Arditti et al. 1980) e altas temperaturas $\left(30^{\circ} \mathrm{C}\right)$ durante o desenvolvimento dos frutos podem prejudicar a viabilidade das sementes, assim como coletas em casa de vegetação, onde pode haver prevalência de sementes sem embriões pela falta do agente polinizador natural (Alvarez-Pardo et al. 2006). Tais fatores podem ter influenciando o elevado número de sementes com embriões abortados encontrados para C. purpurata, principalmente, devido a esta ser a única espécie analisada ocorrente exclusivamente na região sul do Brasil, e, portanto, caracterizada por habitar ambientes com baixas temperaturas quando comparada às demais espécies analisadas.

Em relação as suas dimensões, Orchidaceae apresenta sementes microscópicas, geralmente desprovidas de endosperma, que as tornam dependentes de associações micorrízicas durante a germinação (Smith 1966). Normalmente as sementes do tipo Cymbidium apresentam comprimento entre 0,5 e 1,0 mm (Barthlott \& Ziegler 1981, 
Dressler 1993), entretanto as estudadas neste trabalho apresentaram comprimento médio entre 0,36 e 0,44 mm. Contudo, estas se encontram no intervalo de comprimento apresentado paraorquídeas em geral, ou seja, de 0,15 a 8,0 mm de comprimento (Molvray \& Kores 1995, Pridgeon et al. 1999).

Com relação à largura das sementes, as dimensões encontradas para as espécies estudadas estão de acordo com o intervalo entre 0,06 e 0,24 mm, relatado para espécies de vários gêneros asiáticos de orquídeas (Swamy et al. 2004). A proporção entre comprimento e largura fornece alguns dados relevantes de importância taxonômica (Arditti et al. 1980, Augustine et al. 2001, Swamy et al. 2004). Para as orquídeas analisadas neste trabalho, a proporção apresentou intervalo entre 3,1 e 4,9, ou seja, proporção intermediária às encontradas para outras espécies da família, cujo intervalo constatado foi de 1,73 a 7,41 (Swamy et al. 2004). As dimensões da semente são um reflexo de seu volume, portanto a inferência desse caráter apresenta elevada importância taxonômica (Arditti 1979). O volume seminal médio encontrado nas espécies de Cattleya analisadas apresentou-se entre $0,0006 \mathrm{~mm}^{3}$ e $0,0015 \mathrm{~mm}^{3}$, dentro do intervalo também encontrado para diversas espécies endêmicas da Ásia setentrional, as quais apresentaram volume médio entre $0,0002 \mathrm{~mm}^{3}$ e $0,0118 \mathrm{~mm}^{3}$ (Swamy et al. 2004). Contudo, deve-se salientar que, dentre as espécies analisadas neste trabalho, C. purpurata apresentou o menor volume médio seminal devido à taxa elevada de sementes contendo embriões com baixo vigor, pois a largura do embrião, geralmente exerce influência direta na largura da semente (Arditti 1979).

O estudo detalhado das células da testa das sementes de Orchidaceae pode ser uma das áreas mais promissoras para encontrar características filogeneticamente informativas (Pridgeon et al. 1999). Em relação ao comprimento das células da testa, segundo Vij et al. (1992), sementes de orquídeas podem ser classificadas em três categorias: longas $(>200 \mu \mathrm{m})$, intermediárias $(100-200 \mu \mathrm{m}) \mathrm{e}$ curtas $(<100 \mu \mathrm{m})$; tais categorias, em conjunto com os caracteres dimensões, forma, espessamento das paredes celulares, largura celular e número médio de células, foram utilizadas como parâmetros taxonômicos na identificação de relações filogenéticas em várias Orchidaceae (Healey et al. 1980, Augustine et al. 2001). Por apresentarem-se na categoria sementes de testa longa e pela proximidade dos valores numéricos médios encontrados para as variáveis comprimento, largura e número médio das células da testa, torna-se explícita a similaridade estrutural das espécies analisadas neste estudo, fato esperado devido a órgãos como sementes serem conservativos quanto à morfologia.

Com relação ao volume seminal, salienta-se na literatura que tal caráter apresenta uniformidade genérica (Healey et al. 1980). Para os caracteres proporção do volume seminal/embrionário e porcentagem de espaço aerífero, é comumente relatado que grandes variações podem ocorrer dentro de diferentes populações de uma mesma espécie (Arditti et al. 1980, Augustine et al. 2001).
A presença de espaço aerífero em sementes de orquídeas é importante ecologicamente, pois torna tais diásporos leves e anemocóricos (Arditti et al. 1980, Augustine et al. 2001), e por consequência, aptos a serem dispersos para áreas geográficas distantes, o que facilita a colonização de vários habitats (Augustine et al. 2001), como o ocorrente com as espécies aqui estudadas, com exceção de C. purpurata, que é restrita ao sul do Brasil. Assim, tal caráter assume importância na elucidação de questões não só taxonômicas e filogenéticas, mas também no que diz respeito à fitogeografia (Swamy et al. 2004). O espaço aerífero seminal também se relaciona com o hábito apresentado pelas orquídeas, pois, em análises realizadas com 16 espécies de Liparis Rich, de hábito terrestre, pertencente à Epidendroideae, notou-se que o volume embrionário e a porcentagem de espaço aerífero no interior da semente apresentavam-se correlacionados aos hábitos das plantas. Evidencia-se que espécies epífitas apresentam embriões maiores que as espécies terrícolas e, consequentemente, menor porcentagem de espaço aerífero na semente, fato esse que, possivelmente, afeta o transporte seminal pela via anemocórica e sugere dispersão menos eficiente entre as epífitas, como as espécies analisadas, em comparação às terrícolas (Tsutsumi et al. 2007).

\section{Análises anatômicas e biométricas dos embriões}

Todos os embriões analisados apresentam-se da forma comumente descrita para Orchidaceae, ou seja, uma massa de células sem diferenciação (Smith 1966, Pridgeon et al. 1999); apenas em Vanilla Plum. ex Mill. foi relatada a presença de uma massa de células diferenciadas em três tecidos: protoderme unisseriada, um grupo de 15 células pequenas que constituem o meristema apical caulinar e células parenquimáticas (Batygina et al. 2003).

Coloração embrionária amarelo-claro pode ser encontrada em vários representantes de Orchidaceae (Arditti 1979, Swamy et al. 2004), corroborando os resultados descritos para todas as espécies analisadas.

São escassos na literatura artigos relatando a morfologia embrionária em Orchidaceae. Entretanto, embriões elipsóides, assim como os descritos para C. loddigesii e C. lundii, puderam ser observados para as espécies terrestres Paphiopedilum delenatii Guill e Cypripedium formosanum Hayata, pertencentes à Cypripedioideae (Lee et al. 2005, 2006). A morfologia esférica, assim como a encontrada para C. bicolor, C. purpurata e C. tigrina se apresenta como a mais comumente relatada para os representantes de Laeliinae (Arditti 1979, Clements 1999).

Variações nas dimensões embrionárias são comumente descritas para as Orchidaceae, demonstrando que os caracteres comprimento, largura e proporção comprimento/ largura se apresentam confiáveis para o estabelecimento de relações taxonômicas e filogenéticas (Clements 1999, Pridgeon et al. 1999, Swamy et al. 2004). Tal fato corrobora as variações obtidas em relação aos valores médios encontrados para as variáveis comprimento e proporção comprimento/largura embrionários obtidas neste trabalho. Entretanto, ao analisarmos a variável 
largura embrionária, podemos observar que a maioria das espécies de Cattleya analisadas, com exceção de $C$. purpurata, que apresentou o valor médio mais baixo para a variável, demonstra uniformidade comprovada pela ausência de variação estatística significativa encontrada na comparação de suas médias. C. purpurata possivelmente diferiu das demais devido ao baixo vigor de seus embriões. Uniformidades genéricas dimensionais também foram constatadas em embriões de Platanthera (Orchidoideae) (Healey et al. 1980) e, principalmente, em Bulbophyllum Thouars (Epidendroideae) (Augustine et al. 2001).

Em síntese, os caracteres seminais potencialmente úteis para a taxonomia de Cattleya neste estudo foram a cor seminal e embrionária e a largura, comprimento e volume seminal, os quais evidenciaram diferenças ou semelhanças morfológicas entre algumas espécies. Especificamente, observa-se que a coloração seminal amarelo-ouro e o formato seminal elíptico-afilado diferencia a espécie Cattleya lundii das demais; o formato e média do número de células da testa e do embrião, formato da semente e a proporção comprimento/largura da semente demonstram semelhanças morfológicas entre Cattleya loddigesii e Cattleya lundii; os caracteres formato do embrião, proporção comprimento/largura do embrião, proporção volume seminal/embrionário e porcentagem de espaço aerífero seminal ratificam semelhanças morfológicas entre Cattleya bicolor e Cattleya tigrina.

\section{REFERÊNCIAS}

Alvarez-Pardo, V.M., Ferreira, A.G. \& Nunes, V.F. 2006. Seed disinfestation methods for in vitro cultivation of epiphyte orchids from Southern Brazil. Horticultura Brasileira 24:217-220.

Arditti, J. 1979. Aspects of the physiology of orchids. Advances in Botanical Research 7:421-655.

Arditti, J., Michaud J.D. \& Healey, P.L. 1980. Morphometry of orchid seeds. II. Native California and related species of Calypso, Cephalanthera, Corallorhiza and Epipactis. American Journal of Botany 67:347-365.

Augustine, J.,Yogendra, K. \& Sharma, J. 2001. Orchids of India-II: biodiversity and status of Bulbophyllum Thou., Daya publishing house, New Delhi. 231 p.

Barthlott, W. \& Ziegler, B. 1981. Morphologie der Samenschalenals systematische. Merkmalbeiorchideen. Berichte der Deutschen Botanischen Gesellschaft 94:267-273.

Batygina, T.B., Bragina, E.A. \& Vasilyeva, V.E. 2003. The reproductive system and germination in orchids. Acta Biologica Cracoviensia 45:21-34.

Brasil. 2009. Ministério da Agricultura, Pecuária e Abastecimento. Regras para análise de sementes. Mapa/ACS, Brasília. 339 p.

Bukatsh, F. 1972. Benerkemgemzeirdoppelfarbeingastrablau-safranina. Mikrokosmos 61:255-256.

Buzatto, C.R., Ferreira, P.P.A.,Welker, C.A.D.,Seger, G.D.S.,Hertzog, A. \& Singer, R.B. 2010. O gênero Cattleya Lindl. (Orchidaceae: Laeliinae) no Rio Grande do Sul, Brasil. Revista Brasileira de Biociências 8:388-398.

Carneiro, J.G.A. 1985. Armazenamento de sementes florestais. Associação Brasileira de Tecnologia de Semente, Curitiba. 152 p.

Catling, P.M. \& Catling, V.R. 1991. A synopsis of breeding systems and pollination in North American orchids. Lindleyana 6:187-210.

Chase, M.W. \& Pippen, J.S. 1990. Seed morphology and phylogeny in subtribe Catasetinae (Orchidaceae). Lindleyana 5:126-134.

Clements, M.A. 1999. Embryology. In Genera Orchidacearum, 1 (A.M.
Pridgeon, P.J. Cribb, M.W.Chase, F.N.Rasmussen, eds.). Oxford University Press, Oxford, p. 38-58.

Clifford, H.T. \& Smith, W.K. 1969. Seed morphology and classification of Orchidaceae. Phytomorphology 19:133-139.

Cogniaux, C.A. 1898. Orchidaceae. In Flora Brasiliensis 3 (C.F.P. Martius \& A. G. Eichler, eds.) v. 5, p. 123-135.

Correa, M.P. 1984. Dicionário das plantas úteis do Brasil e das exóticas cultivadas 1. Imprensa Nacional, Rio de Janeiro. 474 p.

Dressler, R.L. 1981. The Orchid: natural history and classification. Harvard University Press, Cambridge. 332p.

1993. Phylogeny and Classification of the Orchid Family. Cambridge University Press, Cambridge. 1112 p.

Frazão, D.A.C., Costa, J.D., Coral, F.J., Azevedo, J.A. \& Figueiredo, F.J.C. 1985. Influência do peso da semente no desenvolvimento e vigor de mudas de cacau (Theobroma cacao L.). Revista de Agricultura 60:2-16.

Healey, P.L., Michaud, J.D. \& Arditti, J. 1980. Morphometry of orchid seeds. III Native California and related species of Goodyera, Piperia, Platanthera and Spiranthes. American Journal of Botany 67:508-518.

Jensen, W.A. 1962. Botanical histochemistry. H. H. Freeman and Co, San Francisco. 408 p.

Jeeja, G. \& Aansari, R. 1994.Taxonomic significance of seed surface morphology in Orchidaceae. Rheedea 4:48-59.

Johansen, D.A. 1940. Plant microtechnique. McGraw Hill Book, New York. 523 p.

Larry, H. 1995. Seed morphology of Hydrangeaceae and its phylogenetic implications. International Journal of Plant Science 156:555-580.

Lee, Y.I., Lee, N.,Yeung, E.C. \& Chung, M.C. 2005. Embryo development of Cypripedium formosanum in relation to seed germination in vitro. Journal of the American Society for Horticultural Science 130:747-753.

Lee, Y.I.,Yeung, E.C., Lee, N. \& Chung, M.C. 2006. Embryo Development in the Lady's Slipper Orchid, Paphiopedilumdelenatii, with Emphasis on the Ultrastructure of the Suspensor. Annals of Botany 98:13111319.

Mathews, J. \& Levins, P.A. 1986. The systematic significance of seed morphology in Portulaca (Portulacaceae) under scanning electron microscopy. Systematic Botany 11:302-308.

Molvray, M. \& Kores, J.P. 1995. Character analysis of the seed coat in Spiranthoideae with special reference to the Diurideae (Orchidaceae). American Journal of Botany 82:1443-1453.

Moraes, M.C. 2014. Estudo biotecnológico, citogenético e molecular em espécies de orquídeas endêmicas da flora brasileira. Dissertação 66f., Universidade Estadual Paulista Júlio de Mesquita Filho, São Paulo.

Ness, B.D. 1989. Seed morphology and taxonomic relationships in Calochotus (Liliaceae). Systematic Botany 14:495-505.

Pabst, G.F.J. \& Dungs, F. 1975. Orchidaceae Brasilienses. Hildesheim, Brucke. 219 p.

Pansarin, E.R. 2003. Biologia reprodutiva e polinização em Epidendrum paniculatum Ruiz \& Pavón (Orchidaceae). Revista Brasileira de Botânica 26:203-211.

Pedroso-de-Moraes, C. 2000. Cultivo de orquídeas. Biblioteca Duse Rüegger Ometto, Uniararas. $132 \mathrm{p}$.

Pedroso-de-Moraes, C., Diogo, J.A., Pedro, N.P., Canabrava, R.I., Martini, G.A.\& Marteline, M.A. 2009a. Desenvolvimento in vitro de Cattleya loddigesii Lindley (Orchidaceae) utilizando fertilizantes comerciais. Revista Brasileira de Biociências 7:67-69.

Pedroso-de-Moraes, C. \& Fontana, C.A.D. 2005. Apontamentos sobre Vanilla planifolia Jacks. ex Andrews. Boletim da Coordenadoria das Associações Orquidófilas do Brasil 60:99-101.

Pedroso-de-Moraes, C., Santos, N.S., Massaro, R., Cordeiro, G.M. \& Souza-Leal, T. 2009b. Desenvolvimento in vitro de Cattleya tigrina A. Richard (Orchidaceae) utilizando fertilizantes comerciais. Ensaios e Ciência 13:57-65.

Pedroso-de-Moraes, C., Souza-Leal, T., Panosso, A.L. \& Souza, M.C. 2012. Efeitos da escarificação química e da concentração de nitrogênio sobre a germinação e o desenvolvimento in vitro de Vanilla planifolia Jack ex Andr. (Orchidaceae: Vanilloideae). Acta Botanica Brasilica 26:714-719.

Pijl, L., Dodson, C.H. 1966. Orchid flowers: their pollination and evolution. University of Miami Press, Florida. 536 p. 
Pridgeon, A.M., Cribb, P.J., Chase, M.W. \& Rasmussen, F.N. 1999. Genera Orchidacearum: general introduction, Apostasioideae, Cypripedioideae. Oxford University Press, Oxford. 608 p.

.2006. Genera Orchidacearum: Epidendroideae (part 1). Oxford University Press, Oxford. 591 p.

2009. Genera Orchidacearum: Epidendroideae (part 2). Oxford University Press, Oxford. 585 p.

Rani, U., Singh, S.G., Gupta, S. \& Garg, V. 1993.Morphometry of orchid seeds in Epidendroideae as revealed by SEM. Advances in Plant Science 6:128-133.

Santana, D.G. \& Ranal, M.A. 2004. Análise da germinação: um enfoque estatístico. Editora da Universidade de Brasília, Brasília. 248 p.

Smith, S.E. 1966. Physiology and ecology of orchid micorrhizal fungi with reference to seedling nutrition. New Phytologist 65:488-499.

Sokal, R.R. \& Rohlf, F.I. 1981. Biometry: the principles and practice of statistics in biological research. W.H. Freeman, San Francisco. 859 p.

Stort, E.F., Braga, P.I.S. \& Storti Filho, A. 2011. Biologia reprodutiva de Cattleya eldorado, uma espécie de Orchidaceae das campinas amazônicas. Acta Amazonica 41:361-368.

Stort, M.N.S. \& Martins, P.S. 1980. Autopolinização e polinização cruzada em algumas espécies do gênero Cattleya (Orchidaceae). Ciência e Cultura 32:1080-1084.

Swamy, K.K., Kumar, H.N.K., Ramakrishna, T.M. \& Ramaswamy, S.N. 2004. Studies on Seed Morphometry of Epiphytic Orchids from
Western Ghats of Karnataka. Taiwania 49:124-140.

Tsutsumi, C., Yukawa, T., Lee, N.S., Lee, C.S. \& Kato, M. 2007. Phylogeny and comparative seed morphology of epiphytic and terrestrial species of Liparis (Orchidaceae) in Japan. Journal of Plant Research 120:405-412.

Van den Berg, C. 2008. New combinations in the genus CattleyaLindl. (Orchidaceae). Neodiversity 3:3-12.

Van den Berg, C. \& Chase, M.W. 2000. Nomenclatural notes on Laeliinae-I. Lindleyana 15:115-119.

2004. A chronological view of Laeliinae taxonomical history. Orchid Digest, 68:226-254.

Van den Berg, C., Higgins, W.E., Dressler, R.L., Whitten, W.M., SotoArenas, M.A., Culham, A. \& Chase, M.W. 2000. A phylogenetic analysis of Laeliinae (Orchidaceae) based on sequence data from internal transcribed spacers (ITS) of nuclear ribosomal DNA. Lindleyana 15:96-114.

Van den Berg, C., Higgins, W.E., Dressler, R.L., Whitten, W.M., SotoArenas, M.A. \& Chase, M.W. 2009. A phylogenetic study of Laeliinae (Orchidaceae) based on combined nuclear and plastid DNA sequences. Annals of Botany 104:417-430.

Vij, S.P., Kaur, P., Kaur, S. \& Kaushal, P.S. 1992.The orchid seeds: taxonomic, evolutionary and functional aspects. The Journal of the Orchid Society of India 6:91-107. 